\title{
Kondo resonance behavior of heavy fermion f-electron materials (invited)
}

\author{
J. W. Allen, ${ }^{\text {a) }}$ G.-H. Gweon, and H. T. Schek \\ Department of Physics, University of Michigan, Ann Arbor, Michigan 48109-1120 \\ L.-Z.Liub) \\ Department of Applied Physics, Yale University, New Haven, Connecticut 06520 \\ L. H. Tjeng ${ }^{\mathrm{c})}$ \\ Department of Physics, University of Michigan, Ann Arbor, Michigan 48109-1120
}

J.-H. Park ${ }^{\mathrm{d})}$

AT\&T Bell Laboratories, Murray Hill, New Jersey 07974

W. P. Ellis

Los Alamos National Laboratory, Los Alamos, New Mexico 87545

C. T. Chen ${ }^{\mathrm{e}}$

AT\&T Bell Laboratories, Murray Hill, New Jersey 07974

O. Gunnarsson, O. Jepsen, and O. K. Andersen

Max-Planck Institut für Festkörperforschung, Heisenbergstrasse 1, D-70569 Stuttgart, Germany

Y. Dalichaouch and M. B. Maple

Department of Physics, University of California at San Diego, La Jolla, California 92093

The Kondo properties of the impurity Anderson model provide a theoretical framework for relating thermodynamic and angle integrated electron spectroscopy data in many heavy fermion materials. We describe the success and the challenges of this approach, summarize a detailed analysis of $\mathrm{CeSi}_{2}$, and give a perspective on the relation to the lattice Anderson model. (C) 2000 American Institute of Physics. [S0021-8979(00)93508-4]

The Kondo behavior of the impurity and lattice versions of the Anderson model underlies much of the thinking about heavy fermion $f$-electron materials. It provides a model scenario for spin fluctuations as an emergent low energy scale property, controlled by high energy scale charge fluctuations, and generating the large heavy fermion mass. The charge fluctuations are the model ingredients. The impurity model describes an $N_{f}$ fold degenerate local $f$-orbital with binding energy $\varepsilon_{f}$ and $f-f$ Coulomb repulsion $U_{f f}$ hybridized by matrix element $V(\varepsilon)$ to conduction band electrons with density of states $\rho(\varepsilon)$. $U_{f f}$ acts to energetically separate valence states $f^{n}$ and $V(\varepsilon)$ enables valence fluctuations through electron transfer with the conduction band. The lattice model envisions a periodic array of such "impurities."

The impurity model applied to the $f$-electron (hole) of $\mathrm{Ce}^{3+}\left(\mathrm{Yb}^{3+}\right)$ provides a useful example of its emergent Kondo properties. ${ }^{1}$ The mixed f/conduction electron ground state is a singlet, and as temperature $T$ decreases, the magnetic susceptibility $\chi(T)$ changes from Curie-like to Paulilike around the Kondo temperature $T_{K}$. For very large $U_{f f}$, one considers $f^{0} / f^{1}$ mixed valence and in the limit $N_{f} \rightarrow \infty$

\footnotetext{
a)Electronic mail: jwallen@umich.edu

${ }^{b)}$ Present address: Department of Biomedical, Yale Medical School, New Haven, CT 06520.

${ }^{c}$ Present address: Solid State Physics Laboratory, University of Groningen, Nijenborgh 4, 9747 AG Groningen, The Netherlands.

${ }^{d}$ Present address: Pohang University of Science and Technology, Pohang 790-784, Kyungbuk, Republic of Korea.

e)Present address: Synchrotron Radiation Research Center, Hsinchu ScienceBased Industrial Park, Hsinchu 30077, Taiwan.
}

and if $\Delta(\varepsilon)=\pi \rho V^{2}$ is constant, then $k T_{K}=E_{F} \exp (-1 / J)$, where the Fermi energy is $E_{F}$ and the Kondo coupling constant is $J=N_{f} \Delta / \pi \varepsilon_{f}$. At $T=0$, the magnetic susceptibility per impurity is given by $\chi(0)=\left(n_{f}\right) C / T_{K}$, where $n_{f}$ is the $f$-state occupation and $C$ is the Curie constant for the ionic ground state. With increasing $T$, as the magnetic moment is "unquenched" and its entropy evolves, there is a $T$-linear contribution $\gamma T$ to the specific heat, with $\gamma=\pi^{2} k_{B} n_{f} / 3 T_{K}$ per impurity. If $T_{K}$ is small, $\gamma$ can be very large, and so this is an important model for the large $\gamma$ values of heavy Fermion materials, which can be viewed as showing an enhanced conduction band mass $m^{*}$.

In the spectrum to add and remove $\mathrm{Ce} f$-electrons below and above the Fermi energy, there are $4 f^{1} \rightarrow 4 f^{0}$ ionization and $4 f^{1} \rightarrow 4 f^{2}$ affinity peaks, roughly at $-\left|\varepsilon_{f}\right|$ and $-\left|\varepsilon_{f}\right|$ $+U_{f f}$, respectively. Associated with the Kondo effect, there is the many-body Kondo (or Suhl-Abrikosov) resonance (KR) near $E_{F}$. The Kondo behavior of the $N_{f}=2$ impurity model was invoked in 1981 to estimate $T_{K}$ values using $\left|\varepsilon_{f}\right|$ and $\Delta$ values inferred from the $4 f^{1} \rightarrow 4 f^{0}$ peak in various Ce materials, ${ }^{2,3}$ and in 1982 to provide a basis for the Kondo volume collapse (KVC) model ${ }^{4}$ of the Ce $\alpha-\gamma$ phase transition. In 1983, a reliable large $N_{f}$ theory was achieved for spectroscopies of the $4 f$ and the $3 d$ (core level) states, ${ }^{5,6}$ enabling the KR to be identified in combined $4 f$ photoemission and inverse photoemission spectra of large $T_{K} \mathrm{Ce}$ materials, ${ }^{7}$ and enabling detailed spectral analysis to begin. ${ }^{8-10}$ The detailed impurity model interpretation of $\mathrm{Ce}$ materials has continued ${ }^{11-13}$ vigorously to the present, with 
steady improvements in theory for $T=0$ (Refs. 1 and 14-17) and $T>0$ (Ref. 18), experiment ${ }^{12,13,19-21}$ and spectrum analysis. $^{22,23}$ Apart from the discussion of the highest $T_{K}$ materials ${ }^{24,25}$ and continuing objections by a single group $^{26-28}$ for small $T_{K}$ materials, the interpretation is widely accepted and used for $\mathrm{Ce}$ materials. For $\mathrm{Yb}$ materials, there are various experimental disagreements and inconsistencies $^{29-32}$ that strongly imply ${ }^{29-31}$ sensitive surface properties affecting photoemission data.

The impurity model inter-relates spectroscopy and transport properties. Model parameters for a material are determined by analyzing photoelectron spectra, as described below. The model susceptibility $\chi(0)$ can then be computed and compared to experimental values, or a model value of $T_{K}$ can be inferred from $\chi(0)$ and compared to values of $T_{K}$ determined from other measurements, e.g., the specific heat $\gamma$ value. As described above, the basic model parameters are $\varepsilon_{f}, V(\varepsilon)$ and $U_{f f}$. It is often important to use a realistic $V(\varepsilon)$ calculated from the local density approximation (LDA) of density functional theory, in which case, the overall magnitude of $V(\varepsilon)$ is adjusted by a factor $\kappa$, i.e., $\kappa V(\varepsilon)$. Reasons why $\kappa \neq 1$ have been discussed in Refs. 22 and 23. An important refinement of the basic model is to include spin-orbit and crystal field splittings of the ionic ground state. $T_{K}$ is extremely sensitive to the ground state degeneracy and in both photoemission spectroscopy (PES) and Bremstrallung isochromat spectroscopy (BIS) spectra the KR displays sidebands away from $E_{F}$ at the energies of $4 f^{1}$ spin-orbit and crystal field splittings. The PES sidebands give observable weight near $E_{F}$ even for extremely small $T_{K}$ materials for which the peak just at $E_{F}$ may be too small to be resolved. This fact, discussed in detail already in Ref. 11, was rediscovered $^{26}$ in the early 90 s and incorrectly cited as evidence against the impurity model. The spin-orbit splitting $\left(\approx 0.28 \mathrm{eV}\right.$ for $\left.\mathrm{Ce}^{3+}\right)$ is known from atomic spectroscopy, and the crystal field splittings are often known from neutron scattering spectra, so these are not generally regarded as adjustable parameters. Thus, $\varepsilon_{f}, \kappa$, and $U_{f f}$ are the key quantities obtained from photoelectron spectra. Note that many combinations of values of $\varepsilon_{f}, \kappa$, and $U_{f f}$ can lead to the same $T_{K}$.

The spectroscopy analysis seeks a simultaneous description of three spectra within the same model. Two of the spectra are the $4 f$ ionization spectrum from angle integrated PES and the $4 f$ affinity spectrum from BIS. The $4 f$ contributions to the total PES and BIS spectra must be separated by exploiting some $4 f$ cross-section photon energy $(h \nu)$ dependence. ${ }^{11}$ For example, using $4 f$ PES cross-section resonances that occur at the $\mathrm{Ce} 4 d \rightarrow 4 f$ and $3 d \rightarrow 4 f$ x-ray absorption edges is called resonant PES (RESPES). ${ }^{33}$ The third spectrum analyzed is the $3 d$ core level spectrum from x-ray photoemission spectroscopy (XPS) [or X-ray absorption spectroscopy (XAS)]. Each spectrum displays multiple peaks whose relative positions and relative integrated weights and also shapes depend on the model parameters. For the two $4 f$ spectra, the rough dependences were described above already. The underlying reason why the $3 d$ spectrum also has multiple peaks corresponding (approximately) to final states with varying numbers of $f$-electrons, $f^{0}, f^{1}$, or $f^{2}$, is that the core hole attraction increases the magnitude of $\varepsilon_{f}$ by an amount $U_{f d}$, thereby perturbing the $4 f$ shell. The $4 f$ PES spectrum is most sensitive to $\varepsilon_{f}$ and $\kappa V(\varepsilon)$. The $4 f$ BIS spectrum gives $U_{f f}$ additionally and is not very sensitive to the detailed $\varepsilon$ dependence of $V(\varepsilon)$. The need for one more parameter $U_{f d}$ to describe the $3 d$ XPS spectrum is more than compensated by the fact the spectrum is very sensitive to $\varepsilon_{f}$, $\kappa$, and $U_{f f}$ and not very sensitive to the detailed $\varepsilon$ dependence of $V(\varepsilon)$. Thus, by seeking the best description of all three spectra simultaneously, the model parameter values are considerably overdetermined. In comparing a model and experimental spectdrum, it is necessary to add by hand to a model spectrum the known experimental resolution and also a lifetime broadening due to mechanisms not included in the Anderson model. Although the lifetime broadenings are adjustable, the values are found to be reasonable relative to what is known from general experience in photoelectron spectroscopy on other kinds of materials.

One further refinement in the spectrum analysis procedure is to account for differing contributions to the spectra from the outermost surface and from the bulk. The angle or kinetic energy dependences of the photoemission probe depth have been exploited to prove experimentally ${ }^{13,19}$ that these contributions are different, and to separate them in analysis. ${ }^{23}$ For example, $3 d \rightarrow 4 f$ RESPES is much more bulk sensitive than $4 d \rightarrow 4 f$ RESPES because the photoelectron kinetic energy is much larger. Similarly, the $3 d$ XPS and $4 f$ BIS spectra are much less surface sensitive than is the $4 f$ PES spectrum determined by using $4 d \rightarrow 4 f$ RESPES. Owing to the reduced number of near neighbor atoms at the surface, the surface layer has a larger $\varepsilon_{f}$ and a smaller $V$ (or $\kappa)$. Thus, the surface $T_{K}$ value is always very small and so for large $T_{K}$ materials the surface contribution acts to decrease the near $E_{F} 4 f$ PES weight of a $4 d \rightarrow 4 f$ RESPES spectrum, relative to what is expected from parameters obtained by analyzing the $3 d$ XPS and $4 f$ BIS spectra. Even for small $T_{K}$ materials, there can be a considerable difference between the surface and bulk contributions in the ionization part of the $4 f$ PES spectrum.

The various points and procedures just summarized have been described fully in the presentation of a detailed analysis $^{23}$ of the spectra of $\alpha$ - and $\gamma$-Ce. The spectroscopic parameters obtained yield $T_{K}$ values and a KVC model $\alpha$ - $\gamma$ phase boundary, ${ }^{34}$ in close agreement with the experiment. The $T$ dependence of the KVC model arises because the model parameters, especially $V(\varepsilon)$ for $\mathrm{Ce}$, are volume dependent, so that the system can adjust $T_{K}$ through a $T$-dependent volume to optimize the Kondo energy and entropy to best minimize its total free energy. The two phases are then distinguished by large $\left(T_{K \alpha} \approx 900 \mathrm{~K}\right)$ and small $\left(T_{K \gamma} \approx 100 \mathrm{~K}\right)$ $T_{K}$ values. Near the critical point in the Ce phase diagram, which can be accessed experimentally in the alloy $\mathrm{Ce}_{0.7} \mathrm{Th}_{0.3}$, a continuous and relatively rapid spectral $T$ dependence results. $^{35}$

There is also an intrinsic and much slower $\ln \left(T / T_{K}\right)$ dependence of the spectra, even for fixed parameters, as calculated using the "noncrossing approximation" (NCA). ${ }^{18}$ The $T$ dependence of the PES weight can be observed only if the features of the KR are fully resolved, because the inte- 

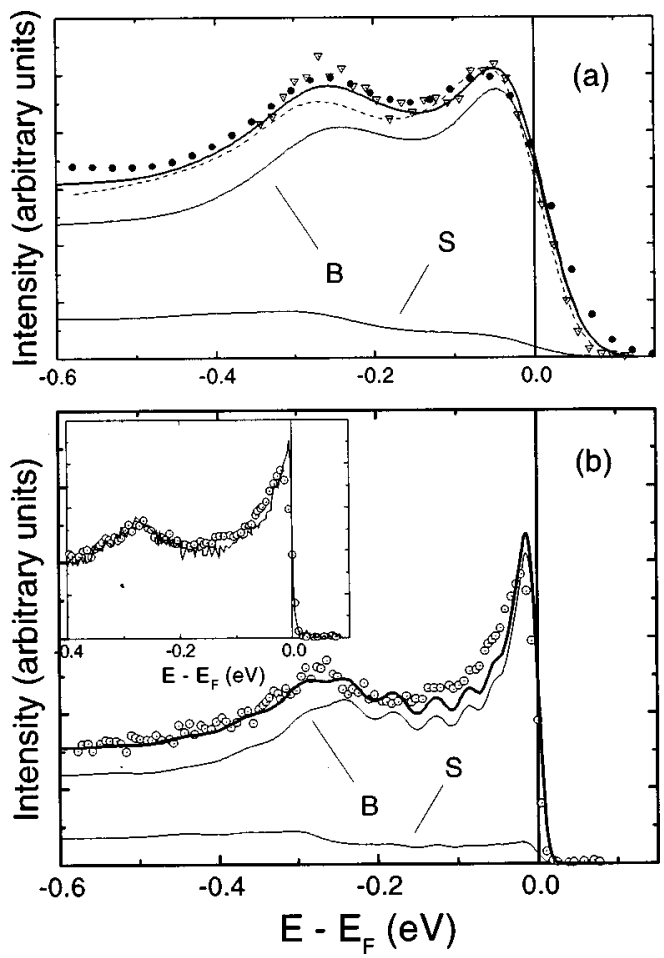

FIG. 1. (a) Comparison of $4 f$ spectra and model description (lines) with surface $(S)$ and bulk $(B)$ components, at medium resolution $(95 \mathrm{meV})$. Our previously unpublished $70 \mathrm{meV}$ resolution spectrum (dots), and $20 \mathrm{meV}$ resolution data (dashes) of Ref. 36 were broadened for comparison to 95 meV resolution data (triangles) of Ref. 26. (b) Same as (a) but for higher resolution $(20 \mathrm{meV})$ data, see Ref. 36. Inset shows 20 and $5 \mathrm{meV}$ (solid line) resolution data from Ref. 40.

grated weight from $E_{F}$ down through the region of the sideband features is only weakly $T$ dependent. ${ }^{1,38}$ Because of the essential asymmetry of $n_{f} \ll N_{f}$, the $T$ dependence of the integrated KR BIS weight is much different and reflects the variation of the thermal average $\left.1-n_{f}(T)\right\rangle$. Since $\left\langle n_{f}(T)\right\rangle$ moves from $n_{f}(0)$ toward 1 for $T \gg T_{K}$, the percentage change in the BIS spectrum can be large. These strongly differing PES and BIS $T$ dependences have been observed. $^{12,20,21,36}$

$\mathrm{CeSi}_{2}$ has a $T_{K}$ value between 35 and $40 \mathrm{~K}$, apparently temperature independent. It has served as a focal point of debate with claims, ${ }^{26}$ reasserted ${ }^{37}$ after challenges, ${ }^{38,39}$ that neither the angle integrated $f$ spectrum nor its $T$ dependence can be described by the impurity model. The observations of the impurity model $T$ dependences of the PES and BIS spectra have been reported in detail elsewhere. ${ }^{20,21,36}$ By paying full attention to all the points described above and by analyzing both $3 d \rightarrow 4 f$ and $4 d \rightarrow 4 f$ RESPES measurements of the $4 f$ PES spectrum, one also can obtain an impurity model description of all the spectra as good as was obtained previously for $\alpha$ - and $\gamma$-Ce. ${ }^{23}$ There is no space in this article to give a detailed description of this analysis and all its results and so we show here only the final result for the near $E_{F} \mathrm{KR}$ and KR sideband part of the spectrum. A full description will be published elsewhere.

Figure 1(a) shows our previously unpublished $100 \mathrm{~K} 4 d$ RESPES spectrum obtained with $\approx 70 \mathrm{meV}$ resolution at the now-defunct Dragon Beamline at the National Synchrotron
Light Source and a $15 \mathrm{~K}$ spectrum ${ }^{36}$ with $20 \mathrm{meV}$ resolution, both Gaussian broadened to a resolution of $95 \mathrm{meV}$ to match a $15 \mathrm{~K}$ near- $E_{F} 4 d$ RESPES spectrum from Ref. 26. The feature near $0.28 \mathrm{eV}$ is the spin-orbit KR sideband. Also shown with the same Gaussian broadening are the model surface and bulk spectra, for which the model parameters are $\left|\varepsilon_{f}\right|=1.9 \mathrm{eV}, U_{f f}=6.1 \mathrm{eV}, \kappa=0.638$ for the bulk, and $\left|\varepsilon_{f}\right|$ $=2.6 \mathrm{eV}, U_{f f}=6.1 \mathrm{eV}, \kappa=0.425$ for the surface, with a full width at half maximum (FWHM) Lorentzian broadening of $0.3|\varepsilon| \mathrm{eV}$. All curves, theoretical and experimental, agree well, showing the theoretically expected $T$ independence of the low-resolution PES spectrum. Figure 1(b) shows that the same theory, Gaussian broadened by only $20 \mathrm{meV}$, describes very well the $15 \mathrm{~K}$ spectrum with $20 \mathrm{meV}$ resolution. The little wiggles in the theory curve reveal an underlying granularity of certain numerical procedures used to make the present calculation valid for our realistic LDA-determined $\Delta(\varepsilon)$ and any value of $U_{f f}$. The inset compares the $20 \mathrm{meV}$ spectrum to a corrected ${ }^{40}$ spectrum $^{21}$ taken with yet higher resolution of $5 \mathrm{meV}$, comparable to $T_{K}$ itself. When Gaussian broadened to $20 \mathrm{meV}$, the latter agrees very well with the former.

To compare the model and experimental values of $T_{K}$, it is essential to include in the model the crystal field splittings of 25 and $48 \mathrm{meV}$, known from neutron scattering. ${ }^{41}$ Since finding $T_{K}$ is much simpler than calculating the $4 f$ spectrum, this can be done while keeping the full realism that was essential for discussing the entire $4 f$ spectrum. For the model with only spin-orbit splitting, $T_{K}=148 \mathrm{~K}$, but adding the crystal field splittings gives $T_{K}=37 \mathrm{~K}$ in excellent agreement with the experimental estimate of 35-40 K. The only effect of including the crystal field splittings in calculating the $4 f$ spectrum would be to shift some weight from the peak just at $E_{F}$ to the region of the crystal field energies, ${ }^{11}$ which would tend to reduce further the already small difference from experiment in Fig. 1(b). An NCA calculation with infinite $U_{f f}$ and unrealistic Gaussian $\Delta(\varepsilon)$, for which the numerical procedures are simpler and permit both the inclusion of crystal field splittings and $T>0$ for the $4 f$ spectrum, gives ${ }^{40}$ a fair description of the near- $E_{F} 5 \mathrm{meV}$ data, including its $T$ dependence, albeit using a slightly different $\left|\varepsilon_{f}\right|$ than found in the detailed analysis leading to Fig. 1.

That lattice effects must occur has been appreciated from the time of the beginning of the impurity model effort, both from theoretical considerations ${ }^{42}$ and from experiments such as the de Haas van Alphen effect ${ }^{43,44}$ showing $f$-electron contributions to the Fermi surface. Therefore one has expected ${ }^{11}$ the $f$-spectral function to be $\mathbf{k}$ dependent. Although there is no rigorous proof, it is theoretically plausible that the $f$-spectral function summed over $\mathbf{k}$ is closely approximated by the impurity $f$-spectral function. The success of the impurity model for describing angle integrated PES spectra is strong empirical evidence that this is so. Indeed, it is often overlooked that by taking $\Delta(\varepsilon)$ from the LDA, the impurity calculation then includes $f$-electrons on all sites, with manybody effects being treated explicitly on one site while the other sites are treated in the LDA. ${ }^{16}$ Perhaps the major question is whether lattice many-body effects dramatically alter the physics, as for example in Nozières' exhaustion picture ${ }^{45}$ 
and in recent numerical results, ${ }^{46}$ both for a simple $N_{f}=2$ Anderson lattice with one or fewer conduction electrons per site. The success of the large $N_{f}$ impurity model and the empirical evidence against a concentration dependence of $T_{K}$ (Refs. 47-50) for Ce materials strongly suggest that exhaustion effects are not important for large $N_{f}$ and realistic numbers of conduction electrons. We also note that recent numerical results for a lattice model support the KVC picture. ${ }^{51}$ It is nonetheless very interesting to apply angle resolved PES (ARPES) to determine the spectral function $\mathbf{k}$ dependence. In spite of some early efforts, ${ }^{52}$ the many serious technical problems are only just now beginning to be overcome using the most recent generation of synchrotrons and ARPES analyzers. ${ }^{53-55}$

This work was supported at University of Michigan by the U.S. DOE under Contract No. DE-FG02-90ER45416 and by the U.S. NSF Grant No. DMR-99-71611; and at UCSD by U.S. NSF Grant No. DMR-97-05454. The NSLS is supported by the U.S. DOE.

${ }^{1} \mathrm{O}$. Gunnarsson and K. Schöhammer, in Handbook on the Physics and Chemistry of Rare Earths, edited by K. A. Gschneidner, L. Eyring, and S. Hüfner (North-Holland, Amsterdam, 1987), Vol. 10, p. 103.

${ }^{2}$ J. W. Allen, S.-J. Oh, I. Lindau, J. Lawrence, L. I. Johansson, and S. B. Hagström, Phys. Rev. Lett. 46, 1100 (1981).

${ }^{3}$ M. Croft, J. H. Weaver, D. J. Peterman, and A. Franciosi, Phys. Rev. Lett. 46, 1104 (1981).

${ }^{4}$ J. W. Allen and R. M. Martin, Phys. Rev. Lett. 49, 1106 (1982).

${ }^{5}$ O. Gunnarsson and K. Schönhammer, Phys. Rev. Lett. 50, 604 (1983).

${ }^{6}$ O. Gunnarsson and K. Schönhammer, Phys. Rev. B 28, 4315 (1983).

${ }^{7}$ J. W. Allen, S.-J. Oh, M. B. Maple, and M. S. Torikachvili, Phys. Rev. B 28, 5347 (1983).

${ }^{8}$ J. C. Fuggle, F. U. Hillebrecht, J.-M. Esteva, R. C. Karnatak, O. Gunnarsson, and K. Schönhammer, Phys. Rev. B 27, 4637 (1983).

${ }^{9}$ J. C. Fuggle, F. U. Hillebrecht, Z. Zolnierek, R. Lässer, Ch. Freiburg, O. Gunnarsson, and K. Schönhammer, Phys. Rev. B 27, 7330 (1983).

${ }^{10}$ O. Gunnarsson, K. Schönhammer, J. C. Fuggle, F. U. Hillebrecht, J.-M. Esteva, R. C. Karnatak, and B. Hillebrand, Phys. Rev. B 28, 7330 (1983).

${ }^{11}$ J. W. Allen, S.-J. Oh, O. Gunnarsson, K. Schönhammer, M. B. Maple, M. S. Torikachvili, and I. Lindau, Adv. Phys. 35, 275 (1986).

${ }^{12}$ D. Malterre, M. Grioni, and Y. Baer, Adv. Phys. 45, 299 (1996).

${ }^{13}$ L. Duò, Surf. Sci. Rep. 32, 233 (1998).

${ }^{14}$ O. Gunnarsson, N. E. Christensen, and O. K. Andersen, J. Magn. Magn. Mater. 76 \& 77, 30 (1988).

${ }^{15}$ O. Gunnarsson and O. Jepsen, Phys. Rev. B 38, 3568 (1988).

${ }^{16}$ O. Gunnarsson O. K. Andersen, O. Jepsen, and J. Zaanen, Phys. Rev. B 39, 1709 (1989).

${ }^{17}$ O. Gunnarsson and K. Schönhammer, Phys. Rev. B 40, 4160 (1989).

${ }^{18}$ N. E. Bickers, D. L. Cox, and J. W. Wilkins, Phys. Rev. B 36, 2036 (1987).

${ }^{19}$ C. Laubschat, E. Weschke, C. Holtz, M. Domke, O. Strebel, and G. Kaindl, Phys. Rev. Lett. 65, 1639 (1990).

${ }^{20}$ M. Grioni, P. Weibel, D. Malterre, and Y. Baer, Phys. Rev. B 55, 2056 (1997).

${ }^{21}$ M. Garnier, K. Breuer, D. Purdie, M. Hengsberger, Y. Baer, and B. Delley, Phys. Rev. Lett. 78, 4127 (1997).

${ }^{22}$ J.-S. Kang, J. W. Allen, O. Gunnarsson, N. E. Christensen, O. K. Andersen, Y. Lassailly, M. B. Maple, and M. S. Torikachvili, Phys. Rev. B 41, $6610(1990)$.
${ }^{23}$ L. Z. Liu, J. W. Allen, O. Gunnarsson, N. E. Christensen, and O. K. Andersen, Phys. Rev. B 45, 8934 (1992).

${ }^{24}$ E. Weschke, C. Laubschat, R. Ecker, A. Hohr, M. Domke, G. Kaindl, L. Severin, and B. Johansson, Phys. Rev. Lett. 69, 1792 (1992).

${ }^{25}$ D. Malterre, M. Grioni, Y. Baer, L. Braicovich, L. Duo, P. Vavassori, G. L. Olcese, E. Weschke. C. Laubschat, A. Hohr, M. Domke, G. Kaindl, L. Severin, and B. Johansson, Phys. Rev. Lett. 73, 2005 (1994).

${ }^{26}$ J. J. Joyce, A. J. Arko, J. Lawrence, P. C. Canfield, Z. Fisk, R. J. Bartlett, and J. D. Thompson, Phys. Rev. Lett. 68, 236 (1992).

${ }^{27}$ A. J. Arko, J. J. Joyce, A. B. Andrews, J. D. Thompson, J. L. Smith, E. Moshopoulou, Z. Fisk, A. A. Menovsky, P. C. Canfield, and C. G. Olson, Physica B 230-232, 16 (1997).

${ }^{28}$ A. J. Arko and J. J. Joyce, Phys. Rev. Lett. 81, 1348 (1998).

${ }^{29}$ J. M. Lawrence, G. H. Kwei, P. C. Canfield, J. G. DeWitt, and A. C. Lawson, Phys. Rev. B 49, 1627 (1994).

${ }^{30}$ J. J. Joyce, A. B. Andrews, A. J. Arko, R. J. Bartlett, R. I. R. Blythe, C. G. Olson, P. J. Benning, P. C. Canfield, and D. M. Poirier, Phys. Rev. B 54, 17515 (1996).

${ }^{31}$ F. Reinert, R. Claessen, G. Nicolay, D. Ehm, S. Hüfner, W. P. Ellis, G. -H. Gweon, J. W. Allen, B. Kindler, and W. Assmus, Phys. Rev. B 58, 12808 (1998).

${ }^{32}$ J. J. Joyce, A. J. Arko, J. L. Sarrao, K. S. Graham, J. Fisk, and P. S. Riseborough, Philos. Mag. B 79, 1 (1999).

${ }^{33}$ J. W. Allen, in Synchrotron Radiation Research--Advances in Surface and Interface Physics, edited by R. Z. Bachrach (Plenum, New York, 1992), Vol. 1, p. 253.

${ }^{34}$ J. W. Allen and L. Z. Liu, Phys. Rev. B 46, 5047 (1992).

${ }^{35}$ J. W. Allen, J.-S. Kang, and S.-J. Oh, J. Magn. Magn. Mater. 63-64, 515 (1987).

${ }^{36}$ D. Malterre, M. Grioni, P. Weibel, B. Dardel, and Y. Baer, Europhys. Lett. 20, 445 (1992).

${ }^{37}$ J. J. Joyce and A. J. Arko, Phys. Rev. Lett. 70, 1182 (1993).

${ }^{38}$ J. W. Allen and O. Gunnarsson, Phys. Rev. Lett. 70, 1180 (1993).

${ }^{39}$ F. Patthey, W.-D. Schneider, M. Grioni, D. Malterre, Y. Baer, and B. Delley, Phys. Rev. Lett. 70, 1179 (1993).

${ }^{40}$ M. Garnier, D. Purdie, K. Breuer, M. Hengsberger, Y. Baer, and B. Delley, Phys. Rev. Lett. 81, 1349 (1998).

${ }^{41}$ R. M. Galera, A. P. Murani, and J. Pierre, Physica B 156 \& 157, 801 (1989).

${ }^{42}$ R. M. Martin, Phys. Rev. Lett. 48, 362 (1982).

${ }^{43}$ W. R. Johanson, G. W. Crabtree, A. S. Edelstein, and O. D. McMasters, Phys. Rev. Lett. 46, 504 (1981).

${ }^{44}$ F. S. Tautz, S. R. Julian, G. J. McMullan, and G. G. Lonzarich, Physica B 206-207, 29 (1995)

${ }^{45}$ P. Nozières, Eur. Phys. J. B 6, 447 (1998).

${ }^{46}$ A. N. Tahvildar-Zadeh, M. Jarrell, and J. K. Freericks, Phys. Rev. Lett. 80, 5168 (1998).

${ }^{47}$ S. D. Bader, N. E. Phillips, M. B. Maple, and C. A. Luengo, Solid State Commun. 16, 1263 (1975).

${ }^{48}$ Y. Onuki, Y. Furukaya, and T. Komatsubara, J. Phys. Soc. Jpn. 53, 2734 (1984).

${ }^{49}$ K. Winzer, Solid State Commun. 16, 521 (1975).

${ }^{50}$ W. Felsch, Z. Phys. B 29, 211 (1978).

${ }^{51}$ C. Huscroft, A. K. McMahan, and R. T. Scalettar, Phys. Rev. Lett. 82, $2342(1999)$

${ }^{52}$ A. B. Andrews, J. J. Joyce, A. J. Arko, J. D. Thompson, J. Tang, J. M. Lawrence, and J. C. Hemminger, Phys. Rev. B 51, 3277 (1995).

${ }^{53}$ H. Kumigashira, S.-H. Yang, T. Yokoya, A. Chainani, T. Takahashi, A. Uesawa, and T. Suzuki, Phys. Rev. B 55, R3344 (1997).

${ }^{54}$ M. Garnier, D. Purdie, K. Breuer, M. Hengsberger, and Y. Baer, Phys. Rev. B 56, R11399 (1997).

${ }^{55}$ J. D. Denlinger, G.-H. Gweon, J. W. Allen, C. G. Olson, Y. Dalichaouch, B.-W. Lee, M. B. Maple, Z. Fisk, P. C. Canfield, and P. A. Armstrong, Physica B (in press). 